\title{
Examining workgroup diversity effects: does playing by the (group-retention) rules help or hinder?
}

\author{
David J. Stanley • Natalie J. Allen • Helen M. Williams • \\ Sarah J. Ross
}

Published online: 7 February 2011

(C) Psychonomic Society, Inc. 2011

\begin{abstract}
Group diversity researchers are often faced with the problem of calculating diversity indices for groups that are incomplete due to participant nonresponse. Because participant nonresponse may attenuate the correlations that are observed between group diversity scores and outcome variables, some researchers use group-retention rules based on within-group response rates. With this approach, only those groups that have a within-group response rate at, or higher than, the rate prescribed by the group-retention rule are retained for subsequent analyses. We conducted two sets of experiments using computer simulations to determine the usefulness of group-retention rules. We found that groupretention rules are not a substitute for a high response rate and may decrease the accuracy of observed relations, and consequently, we advise against their use in diversity research.
\end{abstract}

Keywords Missing data - Diversity · Groups ·

Retention rules

Over the past two decades, researchers and practitioners have shown considerable interest in understanding the impact that workgroup diversity has on group-level process variables (e.g., conflict, communication) and outcome variables (e.g.,

D. J. Stanley $(\bowtie)$

Department of Psychology, University of Guelph,

Guelph, Ontario, N1G 2W1, Canada

e-mail: dstanley@uoguelph.ca

N. J. Allen $\cdot$ S. J. Ross

Department of Psychology, University of Western Ontario,

London, Ontario, Canada

H. M. Williams

School of Business and Economics, Swansea University,

Swansea, Wales, UK viability, innovation, performance). Some theoretical perspectives, such as the information-processing/decision-making approach (Ancona \& Caldwell, 1992; De Dreu \& West, 2001; Gruenfeld, Mannix, Williams, \& Neale, 1996), predict that diversity will enhance processes and outcomes, whereas others, in particular the social identity, self-categorization, and similarity attraction perspectives (e.g., Byrne, 1971; Hogg \& Abrams, 1988; Tajfel \& Turner, 1986), predict that diversity will disrupt processes and impede outcomes. As various reviewers have concluded, the overall pattern of findings examining the effects of diversity is quite mixed (e.g., Horwitz \& Horwitz, 2007; King, Hebl, \& Beal, 2009; Mannix \& Neale, 2005; Riordan, 2001; van Knippenberg, De Dreu, \& Homan, 2004; Williams \& O’Reilly, 1998). In their review, Mannix and Neale argued that these mixed results will only be clarified "by carefully considering moderators such as context, by broadening our view to include new types of diversity such as emotions and networks, and by focusing more carefully on mediating mechanisms" (p. 32). More recently, King et al. expressed a similar sentiment and emphasized the need, in future diversity research, to examine group processes and outcomes with greater specificity.

We agree with these observations, but would add that methodological issues associated with diversity research also need careful scrutiny. Specifically, we contend that clarifying the mixed results within the diversity literature requires attention to how, and from whom, researchers collect the data on which diversity variables are based (e.g., Riordan, 2001), how well researchers' conceptualizations of diversity match their operationalizations of diversity (e.g., Harrison \& Klein, 2007), and how researchers handle the ubiquitous missing-data problem that hampers the accurate assessment of workgroup diversity (e.g., Allen, Stanley, Williams, \& Ross, 2007b; Newman \& Sin, 2009). The latter issue is the focus of the present research; 
specifically, we examine the effectiveness of rules that govern the decision to drop or retain groups based on within-group response rates. We do this in the context of diversity research, where diversity is operationalized using the standard deviation.

\section{Missing-attribute data and diversity-outcome relations}

Researchers have examined workgroup diversity with respect to numerous group member attributes including work-related, psychological, and demographic variables. When attribute data are missing, it is obvious that the group's diversity score, on that attribute, will be distorted. To illustrate, using the standard deviation to calculate the "age diversity" of a group of four 25-year-olds and one 45-year-old, "true score" diversity would be 8.0 . If the 45 -year-old's age was unavailable, however, "observed" diversity would become an inaccurate 0 .

To assess the potential impact of this type of diversity score distortion, Allen et al. (2007b) conducted a series of computer simulations examining the effects of both random and systematic missing-attribute data on true diversityoutcome relations. They demonstrated that even when attribute data are missing at random, observed correlations typically underestimate true correlations. Allen et al.'s work had suggested that missing data are one of many potential sources of measurement error in group diversity scores. Measurement error causes the attenuation of correlationsa well-established principle in individual-level research. Overall, these results suggest that caution be exercised when interpreting any empirical study in which diversity scores are based on incomplete attribute data. Accordingly, Allen et al. (2007b) recommended that researchers obtain high response rates to ensure that complete groups are used to calculate diversity scores.

Obviously, using complete groups is the ideal. But what about when this ideal is not achievable? The diversity literature has yet to settle on a valid methodological approach to the nonresponse challenge. Moreover, for the most part, diversity researchers appear to have paid little attention to the extensive missing-data literature (e.g., Allison, 2002; McKnight, McKnight, Sidani, \& Figueredo, 2007; Newman, 2009; Roth, 1994; Rubin, 1976; Schafer \& Graham, 2002; Switzer \& Roth, 2002). Indeed, group researchers rarely provide detailed information about response rates or about how incomplete groups were handled (Maloney, Johnson, \& Zellmer-Bruhn, 2010). It appears, however, that the most commonly used approach to mitigating problems associated with nonresponse in diversity research involves the application of various rules governing the decision to drop or retain groups. Of these, some researchers who encounter missing data opt to include only those groups with some minimum number of respondents (e.g., $\geq 2$ per group: Schippers, den Hartog, Koopman, \& Wienk, 2003). More commonly, however, researchers who use group-retention rules base them on within-group response rates, requiring a specific minimum percentage of respondents within each group in order for that group to be retained. Interestingly, the minimum percentage that researchers require varies considerably (e.g., 40\%: Mayo, Pastor, \& Meindl, 1996; 66\%: Reagans, Zuckerman, \& McEvily, 2004; 100\%: Jehn, Northcraft, \& Neale, 1999). Given the use of such rules, and the relatively wide "minimum percentage" range that is observed in the literature, an important question for diversity researchers is: What types of group-retention rules are effective? To put this question into the most relevant research context, we turn to the missing-data literature.

\section{Approaches to missing data}

Although there are many ways to approach missing data (see Allison, 2002; McKnight et al., 2007; Newman, 2009; Roth, 1994; Rubin, 1976; Schafer \& Graham, 2002; Switzer \& Roth, 2002), they generally fall into three categories of procedures: deletion, augmentation, and replacement (McKnight et al., 2007). The underlying goal of these procedures is to provide accurate estimates of underlying population parameters - though some do so more effectively than others (Schafer \& Graham, 2002). Deletion procedures (e.g., listwise, pairwise) typically focus on conducting analyses in the usual way but by using a subset of the data that would be obtained with a $100 \%$ response rate. In contrast, augmentation procedures (e.g., maximum likelihood, expectation maximization, dummy coding adjustments) focus on estimating parameters using the available data in combination with probability models. Finally, replacement strategies attempt to replace missing values with estimates of those values (e.g., single imputation procedures, multiple imputation procedures) as a means of accurately estimating parameters. Of these strategies, the expectation maximization (a type of data augmentation) and multiple imputation (a type of data replacement) procedures tend to produce relatively accurate results (Switzer \& Roth, 2002).

As previously noted, within the diversity literature, the most common approach to handling missing data is groupretention rules. Given the ubiquity of this approach, we believe it is important to examine its effectivenessparticularly since group-retention rules could be considered a deletion procedure. Consider for a moment the logic behind the use of group-retention rules in the typical 
diversity study. Imagine, for example, a diversity research scenario in which the researcher discovers that there is an overall response rate of $50 \%$. The researcher recognizes that participant nonresponse introduces error into the measurement of diversity scores. Recognizing that better data are provided when the response rate is higher, the researcher attempts to simulate a higher response rate by using only those groups with a within-group response rate higher than some retention rule (e.g., 70\%). By doing this, the researcher essentially "drops" or "filters out" those groups that are most contaminated by the measurement error created by participant nonresponse. The apparent logic behind this approach is that it is better to have no information from a group than to have information that is contaminated by high levels of measurement error.

Although group-retention rules are used extensively by diversity researchers, the impact of these rules on the accuracy of diversity findings has never been examined. Consequently, we conducted two sets of experiments using computer simulations to investigate this issue and, on the basis of our findings, offer empirically derived advice for diversity researchers regarding the use of such rules.

\section{Research overview}

The computer simulations we conducted were designed to ask "What happens if . . . ?" under several different participant nonresponse scenarios. Specifically, for each nonresponse scenario, we manipulated the strength of the true diversity-outcome correlation and the group-retention rule. This experimental design allowed us to examine the usefulness of group-retention rules when assessing diversity correlations of varying strengths.

Our simulations examined diversity with respect to a continuous variable, and we operationalized diversity using the standard deviation (with $N$ in the denominator). This operationalization of diversity is consistent with the conceptualization of diversity referred to by Harrison and Klein (2007) as "separation." Merely to provide a context in which to think about diversity, we refer to our simulations as modeling "age diversity." The simulations are equally applicable, however, to any continuous variable (e.g., tenure, values, attitudes, personality).

Two studies were conducted in which we examined different forms of missing data. Using the terminology suggested by Rubin (1976; Little \& Rubin, 2002), in Study 1 we examined the impact of group-retention rules when data were missing randomly (i.e., missing completely at random). In contrast, in Study 2 we examined the impact of group-retention rules when data were missing systematically (i.e., missing not at random).
Study 1: random participant nonresponse

Method

\section{Simulation overview}

1. Creation of groups We created a set of 100 "groups." Specifically, we created 100 groups that varied in size from 5 to 15 "individuals" $(M=10, S D=2.01 ; 1,000$ individuals distributed across 100 groups). This range is typical in group diversity research (Allen et al., 2007b). Group sizes were created using a normal distribution of group sizes.

The individuals in these groups varied with respect to a continuous variable. As we noted earlier, although this variable could represent any one of several continuous variables (e.g., personality, tenure), to enhance clarity we will refer to it as "age." The ages of the individuals composing the groups were randomly sampled from a normal distribution $(M=40, S D=6.6)$.

2. Calculation of true diversity scores Diversity scores were calculated using standard deviation $(N$ in the denominator) for each of the 100 groups using the ages of all group members (i.e., a 100\% response rate).

3. Creation of outcome variables/calculation of true correlations Six outcome variables were created for each group. We conceptualized these outcome variables as constructs that are measured at the group level (e.g., group performance). These six outcome variables were created such that they correlated with the true diversity scores at $.10, .20, .30, .40, .50$, and .60 . We refer to the correlation between the true diversity scores and these outcome variables as "true correlations." The outcome variables were created by combining the true diversity score column with random (normal) error. The random (normal) error column was weighted to create an outcome variable that correlated with the true diversity score column at a magnitude consistent with each true correlation modeled $( \pm .01)$.

4. Creation of response rate scenarios In their review, Allen et al. (2007b) found that the response rates in diversity studies ranged from $46 \%$ to $100 \%$. Consequently, we simulated $30 \%, 50 \%, 70 \%$, and $90 \%$ response rates. To simulate a particular response rate, individuals were randomly removed (i.e., identified as nonresponders) without reference to group membership. Consequently, each individual had an equal probability of being removed (i.e., individuals were missing completely at random). This resulted in a random number of people being removed from each group. Because we modeled overall response rates, it was possible within a set of 100 groups to have a 
variety of within-group response rates. For example, if the response rate was $50 \%$, it was possible for Group 1 to have a $60 \%$ response rate, Group 2 to have a $100 \%$ response rate, Group 3 to have a $0 \%$ response rate, and so on. We examined variability in within-group response rates across replications in the simulation and found that it was similar across replications for a given response rate.

5. Implementation of group-retention rules We examined the impact of 11 group-retention rules. The first level of the group-retention rule variable was a "no rule" groupretention rule. Specifically, under the "no rule" groupretention rule, all groups for which it was possible to calculate a diversity index (i.e., all groups with two or more individuals) were retained for subsequent analyses. This was done to provide a baseline to assist with the interpretation of results and reflects a rule that is sometimes used by diversity researchers. The remaining 10 levels of the group-retention rule variable were percent-based group-retention rules that ranged, in $10 \%$ increments, from $10 \%$ to $100 \%$. To be clear, a " $10 \%$ group-retention rule" means that a group was only retained in the analysis if at least $10 \%$ of its members responded.

6. Calculation of observed diversity scores In groups that were "retained," diversity scores were calculated based only on those individuals who were responders.

7. Calculation of observed correlations The correlation between the observed diversity scores and each outcome measure was calculated.

8. Replications This entire process was repeated 5,000 times. For each replication, we created a new set of simulated individuals that were distributed across the 100 groups, but we ensured that the underlying true correlations were the same across replications (see Step 3 above).

Results and discussion

\section{Overview of analytic strategy and interpretation of figures}

In order to evaluate the impact of the various retention rules that we applied, we examined (a) the number of groups that were retained and (b) the accuracy with which the observed correlations estimated the true correlations.

Bias and variability With respect to point (b), it is important to note that the accuracy with which a parameter is estimated is typically evaluated with respect to two criteria: variability and bias (Dekking, Kraaikamp, Lopuhaa, \& Meester, 2005). Interpreting variability (i.e., efficiency) and bias concurrently, however, can be difficult. In the case under consideration here, for example, if retention rules decrease bias, but increase variability, it would be difficult to determine the overall effect of a retention rule on the accuracy of estimates of a relation. Thus, we used mean absolute error as a means of measuring the combined influence of bias and variability. The analogy of shooting a target may help to understand how mean absolute error is calculated. Consider a shooter who aims at the center of the target. The shooter hits the target with a very narrow cluster of shots (i.e., low variability), the center of which is three inches below the center of the target (i.e., high bias). Now consider a second shooter who hits the target with a very wide cluster of shots (i.e., high variability), the center of which is only a half-inch below the center of the target (i.e., low bias). If you were interested in picking the more accurate shooter, who would it be? In this example, you might use, for each shooter, the distance between each shot that hit the target and the center of the target. You could then obtain an average accuracy for each shooter that would reflect both bias and variability. We use the term "mean absolute error" to refer to an index that concurrently assesses the influence of both bias and variability on the accuracy of relations. A low mean absolute error value indicates both low bias and low variability. A high mean absolute error value could occur due to high bias, high variability, or both.

Interpreting the figures Results for the random nonresponse simulations conducted in Study 1 are illustrated in Figs. 1 and 2. Figure 1 illustrates the number of groups retained, whereas Figure 2 illustrates the bias, variability, and mean absolute error for each response rate. These results are somewhat easier to understand by considering the response rate; thus, on each graph shown in the figures, we placed a solid vertical line extended off the $x$-axis at the level of the response rate (e.g., for a $30 \%$ response rate, there is a vertical line extending up from the $30 \%$ retention rule). This line is helpful, because it illustrates the impact of the various group-retention rules relative to the response rate. Specifically, readers can easily see the impact of using group-retention rules that are larger (or smaller) than the response rate by comparing the pattern of results on either side of this vertical line.

\section{Number of groups retained}

The graphs shown in Fig. 1 illustrate the number of groups retained using each of the group-retention rules for a given response rate. The $x$-axis indicates the group-retention rule, whereas the $y$-axis indicates the number of groups retained. As is obvious, and can be seen on the left side of each of graph, the "no rule" (NR) group-retention rule results in 
Fig. 1 Study 1: random nonresponse. Number of groups retained for each retention rule
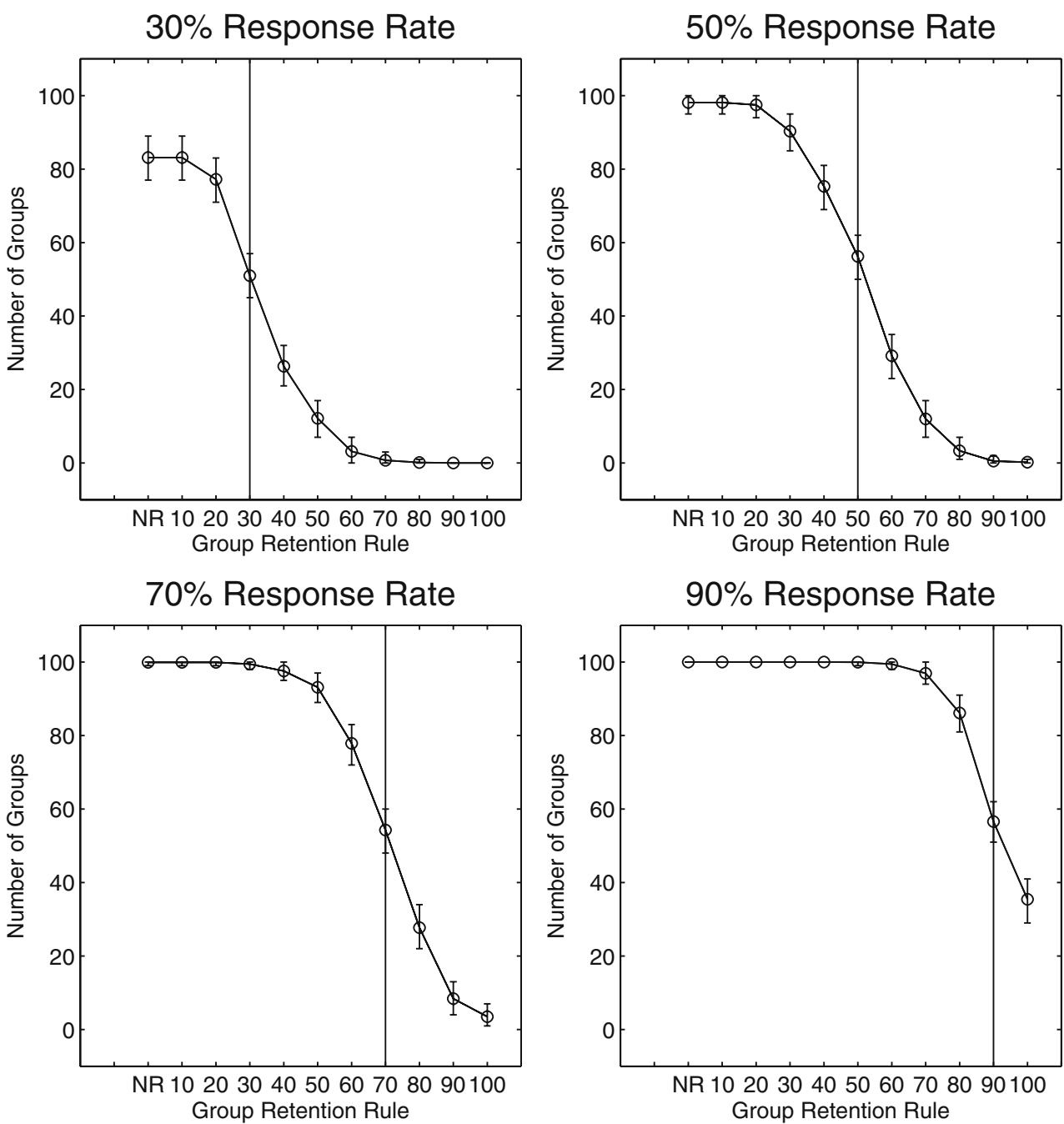

nearly $100 \%$ group retention. ${ }^{1}$ Beyond this, due to differences in the composition of groups across replications, different numbers of groups were retained in each of the 5,000 replications. The graph illustrates this fact in two ways. First, each dot on this graph represents, averaging over the 5,000 replications, the mean number of groups (of the 100) that are retained using each group-retention rule. Second, the variability in the number of groups retained across the 5,000 replications is illustrated with the range whiskers around each dot. These range whiskers were constructed empirically by rank ordering the number of groups retained (for a given group-retention rule) in each of the 5,000 replications and then choosing end points for the range that bounded the middle $95 \%$ of these values.

An inspection of the graphs in Fig. 1 reveals that the number of groups retained varied with different groupretention rules. Not surprisingly, high group-retention rules

\footnotetext{
${ }^{1}$ Some groups were dropped under the "no rule" group-retention rule because they had fewer than two respondents.
}

resulted in fewer groups being retained. Importantly, as we will illustrate below, retaining fewer groups increases mean absolute error by increasing the variability in observed correlations. The number of groups retained also depended, however, on the response rate. Using a retention rule that required a within-group response rate higher than the overall response rate (represented by the solid vertical line) resulted in a large number of groups being dropped from analyses. For example, in the Fig. 1: 50\% response rate graph, when a group-retention rule of $30 \%$ was used, on average, 90.30 of the 100 groups per replication were retained. In contrast, when a $70 \%$ group-retention rule was used (i.e., a rule higher than the response rate), an average of only 11.98 of the 100 groups per organization were retained.

\section{Mean absolute error}

Because interpreting both bias and variability information can be difficult, we created the "Mean Absolute Error" 
30\% Response Rate

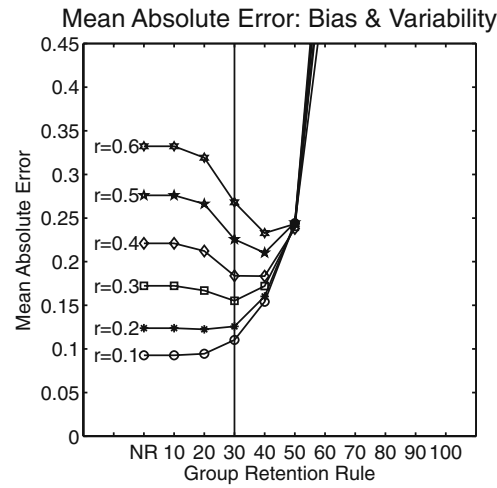

$50 \%$ Response Rate

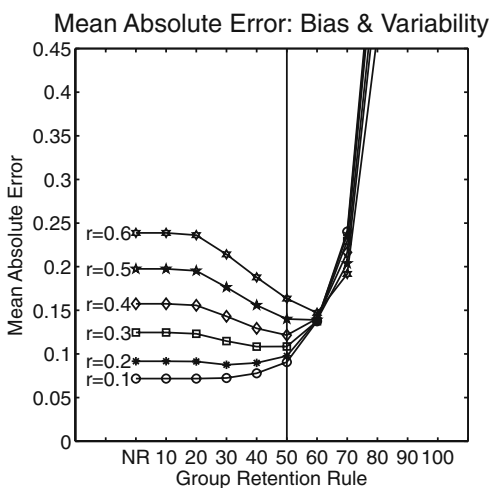

\section{0\% Response Rate}

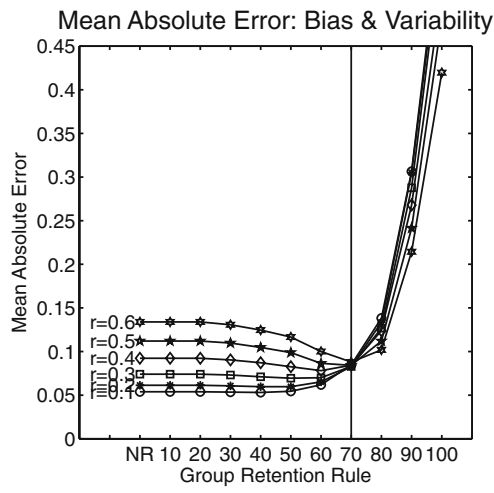

\section{0\% Response Rate}

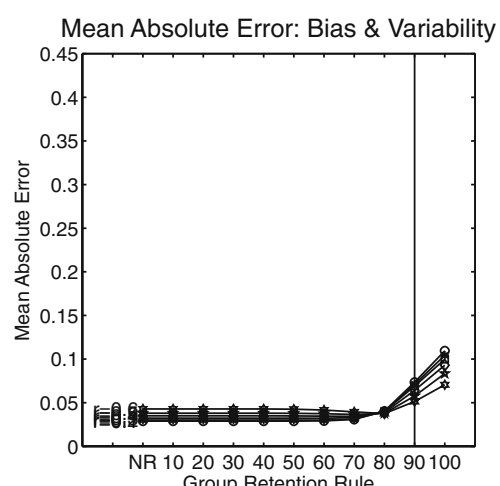

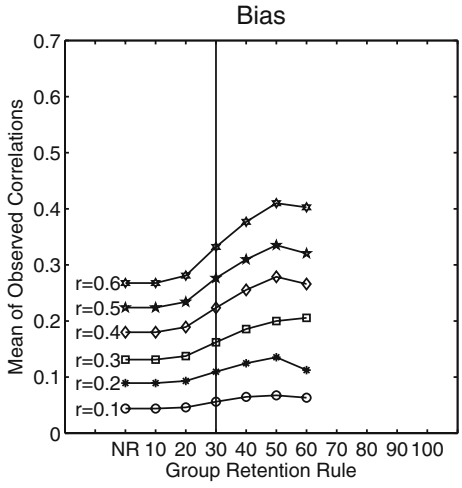
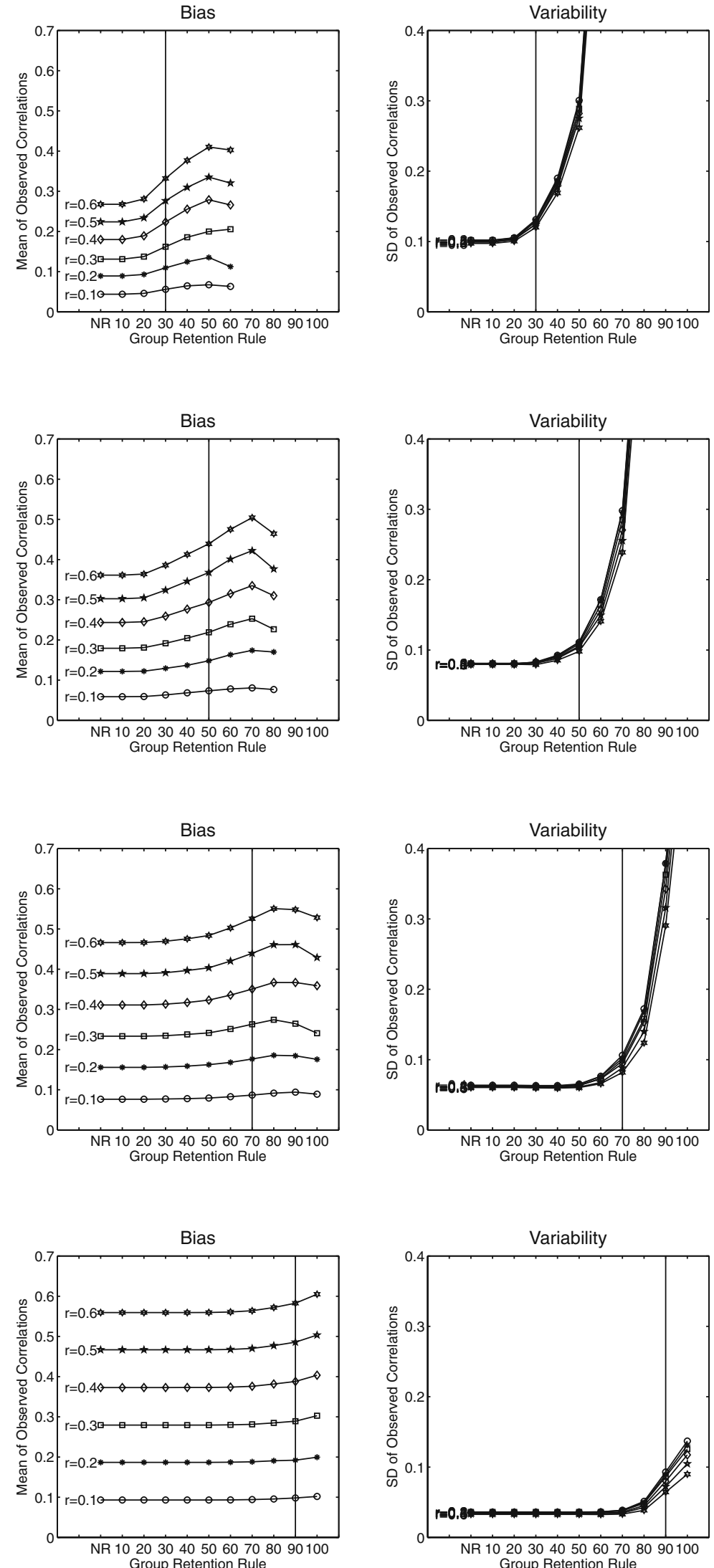

NR 1020304050607080

Fig. 2 Study 1: random nonresponse. Mean absolute error, bias, and variability for each retention rule across various response rates 
graphs (see Fig. 2) using the logic described above. The mean absolute error values in these graphs were calculated using Eq. 1 below ( $K$ represents the number of replications) - a modified version of the absolute error approach used by Roth, Switzer, and Switzer (1999). We took the absolute value of the difference between $r_{\text {observed }}$ and $r_{\text {true }}$, whereas Roth et al. squared this difference.

Mean Absolute Error $=\frac{\sum_{1}^{K}\left|r_{\text {observed }}-r_{\text {true }}\right|}{K}$

As can be seen in Fig. 2, in no case did group-retention rules fully compensate for participant nonresponse. Indeed, in all circumstances, mean absolute error was lower (i.e., accuracy was higher) for higher response rates. For example, as the mean absolute error graphs illustrate, regardless of the retention rule used, mean absolute error was lower for all true correlation lines in the $50 \%$ response rate graph than their counterparts in the $30 \%$ response rate graph.

Increasing the within-group response rate required for retention influenced mean absolute error differently for strong and weak true correlations. Consider, for example, the simulation with a $30 \%$ response rate in Fig. 2. Increasing the within-group response rate required for retention (i.e., moving left to right along the $x$-axis in the mean absolute error figure) decreased the error associated with estimates of strong true correlations (i.e., true correlation in the range of .40 to .60) but not for weak true correlations (i.e., true correlation less than .40). Moreover, increasing the within-group response rate required for retention had little effect on, or actually increased, the error associated with estimates of weak true correlations. This pattern of relations was true for low response rates, but less so for high response rates. Indeed, a comparison of the mean absolute error graphs for the $30 \%$ and the $90 \%$ response rates reveals that retention rules had (for the most part) little impact on error when the response rate was high. Notably, however, using a $90 \%$ or $100 \%$ retention rule with a $90 \%$ response rate increased the error associated with estimates of true relations.

When a group-retention rule was substantially lower than the response rate, the rule did little to change the accuracy (i.e., mean absolute error) of estimates. Rules had little effect in this situation because few, or none, of the groups were dropped. It was only when the group-retention rule approached the response rate, and approximately 50\% of groups were dropped, that the accuracy of estimates was influenced. Moreover, when the retention rule exceeded the response rate (i.e., to the right of the vertical line in each graph), even fewer groups were retained. The consequence of retaining so few groups was a substantial increase in mean absolute error (reflected in the sharp upward curves in the mean absolute error graphs).

Why did these changes in mean absolute error occur? Mean absolute error changes as a function of the amount of bias and variability created by missing data. In the next two sections, we outline how the changes in mean absolute error were a direct consequence of changes in bias and variability.

\section{Bias}

In Fig. 2, the graphs labeled "Bias" illustrate, for a given response rate, the attenuation of observed correlations that occurred using the various group-retention rules that we modeled. The $x$-axis indicates these group-retention rules. The $y$-axis indicates the mean of the observed correlations for groups that conformed to the retention rule. Each line on the bias graphs represents a different true correlation, and each point on these lines represents the mean of the observed correlations across the 5,000 replications (i.e., mean observed correlation) for a particular group-retention rule. To the extent that the mean observed correlation does not correspond to the true correlation (indicated by the line label-e.g., $r=.60$ ), the observed relation is a biased estimate of the true relation.

As can be seen in Fig. 2, the observed correlations are underestimates of true correlations, indicating varying degrees of bias. Moreover, increasing the within-group response rate that was required for retention decreased bias (to a point), but did so differentially for strong and weak true correlations. The Fig. 2: 50\% response rate bias graph, for example, illustrates this finding. Increasing the withingroup response rate required for retention increased the mean observed correlations (i.e., decreased bias) to a greater extent for a .60 true correlation than for a .10 true correlation. Specifically, for the .60 true correlation, when no retention rule was used the mean observed correlation was .36; however, when a $50 \%$ retention rule (equal to the response rate) was used, the mean observed correlation was .43 (i.e., closer to the true correlation of .60-hence, less biased). In contrast, for the .10 true correlation, when no retention rule was used, the mean observed correlation was .06; however, when a $50 \%$ retention rule (equal to the response rate) was used, the mean observed correlation was .074 (i.e., closer to the true correlation of .10-hence, less biased). Interestingly, when the within-group response rate required for retention was slightly higher than the response rate, bias started to increase again (represented by the line going down) - likely due to the extremely high number of groups that were dropped (see Fig. 1).

Estimates of strong and weak true correlations were differentially biased because the measurement error created 
by nonresponse influences them differently. Specifically, the influence of measurement error is proportional to the strength of the true correlation (as indicated by the classic Spearman, 1910, attenuation formula, in which the true correlation is multiplied by the square root of the index of measurement error [i.e., reliability] to obtain the mean observed correlation). Consequently, changes in measurement error (i.e., reliability) associated with diversity scores will influence strong correlations to a greater absolute extent than weak correlations. Group-retention rules drop groups with low within-group response rates (i.e., those high in measurement error) and retain groups with higher within-group response rates (i.e., those low in measurement error). As a result, the observed correlations are typically less biased when group-retention rules are used. Moreover, because measurement error acts in a manner proportional to the strength of the true correlation, the absolute decrease in bias is greater for strong true correlations.

\section{Variability}

In Fig. 2, the graphs labeled "Variability" illustrate, for a given response rate, the variability in observed correlations that occurred using the various group-retention rules that we modeled. The $x$-axis indicates group-retention rules. The $y$-axis indicates the standard deviation of the observed correlations based on groups that passed the retention rule. Each line on this graph represents a different true correlation, and each point on these lines represents the standard deviation of the observed correlations averaged across the 5,000 replications for a particular group-retention rule.

The variability graphs reveal the sharp increase in variability, in observed correlations, that occurs when the within-group response rate required for retention exceeds the overall response rate. Further, it can be seen in Fig. 2 that, although retention rules influenced the bias associated with strong and weak correlations differently, this was not the case with respect to variability. Indeed, inspection of the variability graphs reveals that increasing the within-group response rate required for retention had a similar effect on the variability of observed correlations for strong and weak true correlations. Specifically, increasing the within-group response rate required for retention dropped more groups from the analysis and increased the standard deviation of the observed correlations. Thus, although using retention rules decreased the bias associated with estimates, it increased the variability associated with estimates. Moreover, when the within-group response rate required for retention was higher than the response rate, the variability in observed correlations increased considerably. This increase in variability substantially increased mean absolute error when the retention rule was the same as, or slightly higher than, the response rate.
Summary

A key observation from the results of Study 1 is that the influence of group-retention rules is complex. Strikingly, we found that group-retention rules were not able to compensate for a low response rate. That is, using groupretention rules did not increase the accuracy of estimates obtained at one response rate (e.g., 50\%) to equal those obtained at a higher response rate (e.g., $70 \%$ or $90 \%$ ). The extent to which retention rules could be used to increase the accuracy of estimates depends on the balance between the changes in bias and variability created by the rules. Using a retention rule that required a high within-group response rate decreased the bias associated with estimates (to a point), but simultaneously increased the variability associated with estimates. When using such a rule, the decrease in bias was greater for strong than for weak true correlations. The increase in variability, however, was similar for all true correlations. Consequently, the greater decrease in bias associated with strong correlations resulted in retention rules being more effective for strong true correlations. Generally, for strong true correlations (.40 and above), when the retention rule was equal to (or less than) the response rate, the decrease in bias was greater than the increase in variability, and accuracy increased (i.e., mean absolute error decreased). In contrast, for weak true correlations (below .40), the variability in observed correlations was always greater than the decrease in bias. Consequently, for weak true correlations (i.e., those typically observed by diversity researchers), retention rules either had little effect or decreased the accuracy of estimates of relations. $^{2}$

\section{Study 2: systematic participant nonresponse}

In the simulations described in Study 1, we focused on the impact of using group-retention rules when data were missing randomly. In the simulations presented in Study 2, we examine the impact of using group-retention rules when data were missing systematically. The latter approach is consistent with recent research that has suggested that nonresponse is often linearly related to the variables investigated (e.g., Rogelberg, Conway, Sederburg, Spitzmüller, Aziz, \& Knight, 2003; Rogelberg, Luong, Sederburg, \& Cristol, 2000).

Consequently, in the linear nonresponse simulations we modeled nonresponse such that individuals who were young (relative to the ages of all others in the replication) were less likely to respond. Additionally, we also modeled nonresponse that is based on the relations of nonresponders

\footnotetext{
${ }^{2}$ We also conducted simulations in which group size was held constant. The overall pattern of results, with respect to the impact of retention rules, was similar.
} 
with other members of their group (rather than the sample as a whole). This approach is consistent with other simulation research in this area (e.g., Allen, Stanley, Williams, and Ross 2007a) and, importantly, takes into account the well-established view that group context influences individual behavior (Hackman, 1992). More specifically, it is consistent with theorizing, and empirical evidence, within the diversity and relational demography literatures, that group members are sensitive to their relative position within the group and that dissimilarity/similarity on various attributes can shape behavior (e.g., Jackson et al., 1991; Liao, Joshi, \& Chuang, 2004; O’Reilly, Caldwell, \& Barnett, 1989). Moreover, although little empirical attention has been paid to the possibility that one's relative position within the group can influence the specific behavior modeled here (i.e., nonresponse behavior), Allen et al. (2007a) reported data from a publicly funded agency in the United Kingdom in which individual scores on an attribute, relative to others in their workgroup, were related to nonresponse. Thus, in addition to the linear nonresponse simulations, described above, we modeled a linear nonresponse (within-group one-tail) condition. ${ }^{3}$ In the linear nonresponse (within-group one-tail) condition, younger individuals within each group had a higher probability of being nonresponders.

\section{Method}

\section{Simulation overview}

\section{Groups, diversity scores, and outcome variables/true} correlations This simulation used a process similar to Study 1 to create groups, diversity scores, and outcome variables/true correlations (see Steps 1-3 in Study 1).

2. Creation of response rate scenarios As in Study 1, we simulated $30 \%, 50 \%, 70 \%$, and $90 \%$ response rates. In contrast, however, we modeled two types of systematic nonresponse instead of random nonresponse. These are outlined below.

Linear nonresponse was modeled in a manner similar to past research (see Newman, 2003; Newman \& Sin, 2009; Switzer, Roth, \& Switzer, 1998). In the context of the present simulations, that means that, of all the individuals in a particular replication, younger individuals were more likely to be nonresponders. The ages of all individuals within a replication were

\footnotetext{
${ }^{3}$ In addition to these two forms of systematic nonresponse, we also conducted simulations use the probabilistic implementations of two nonlinear nonresponse scenarios from Allen et al. (2007b). Specifically, in the nonlinear nonresponse (within-group two-tail) scenario, the "oldest" and "youngest" within the group were most likely to be missing. In contrast, in the nonlinear nonresponse (within-group median) scenarios, individuals whose age was at the middle of the within-group age distribution were most likely to be missing.
}

linearly transformed to a "probability-of-deletion" score. Next, these probability-of-deletion scores were added to a random (uniform) variable to create a "cut score" variable such that younger individuals were more likely to be nonrespondents than older individuals within the replication. The strength of the systematic nonresponse can be indexed by the relation between group probability of deletion scores and cut scores. In our simulations, this was set to $d=0.45$ (i.e., in the middle of the 0.30 to 0.60 range used by Newman \& Sin, 2009). Individuals were rank ordered on these cut scores and then deleted in a top-down fashion, such that younger individuals within a replication were more likely to be designated as nonresponders.

Linear nonresponse (within-group one-tail) was modeled such that the younger individuals in each group had a higher probability of being nonresponders. This was accomplished using a variation of the linear nonresponse procedure described above. First, we determined the number of individuals that would be missing from each group if a "missing completely at random" deletion procedure had been used. Individuals were not deleted completely at random; we simply determined the number of individuals that would have been deleted from each group for a given response rate using this procedure. This was done so that the number of individuals that were designated as nonresponders within each group was randomly determined. Second, we selected the specific individuals that were to be designated as nonresponders. In the present context, however, linear nonresponse occurred within each group such that the younger individuals within each group were more likely to be nonresponders.

3. Retention rules, observed diversity scores, observed correlations, and replications The rest of the simulation paralleled Study 1 with respect to the implementation of retention rules, calculation of observed diversity scores, calculation of observed correlations, and replications (see Steps 5-8 in Study 1).

\section{Results}

The results for the linear and linear (within-group one-tail) nonresponse simulations are presented in Figs. 3 and 4, respectively. The "Number of Groups Retained" graphs are not presented for Study 2 because they were nearly identical to those presented in Study 1 for both forms of systematic nonresponse. ${ }^{4}$

\footnotetext{
${ }^{4}$ We do not report the sizes of the retained groups because it is the number of individuals missing from each group that dictates whether a group is dropped or retained - not the specific individuals that are missing (i.e., the type of nonresponse: random or systematic).
} 


\section{0\% Response Rate}

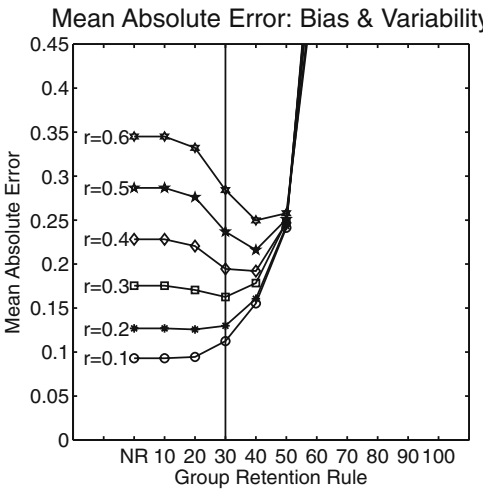

\section{0\% Response Rate}

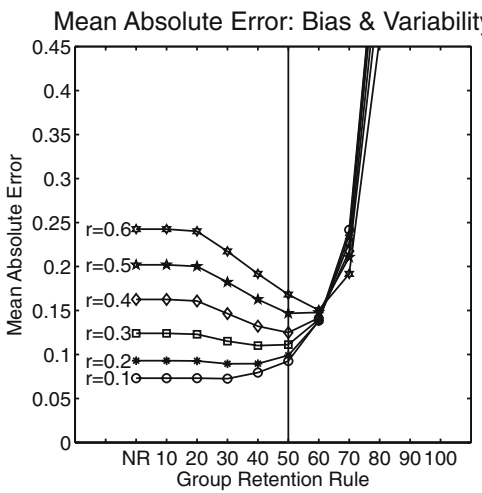

70\% Response Rate

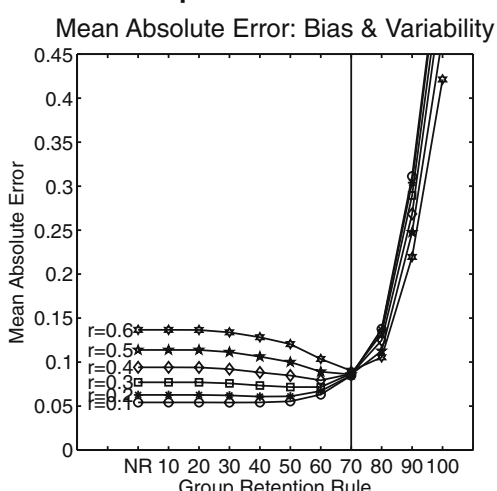

Group Retention Rule

\section{0\% Response Rate}

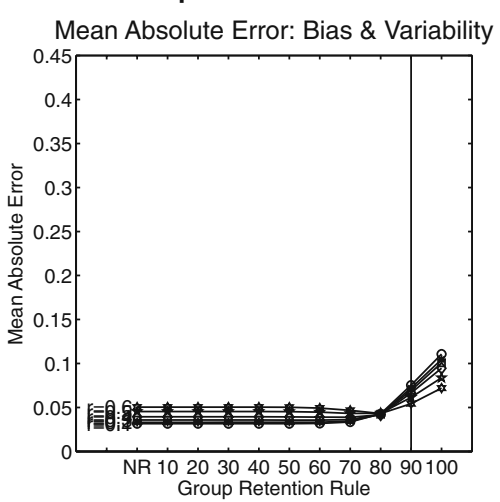

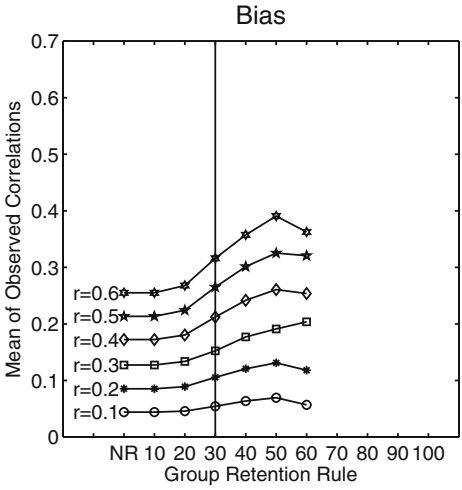
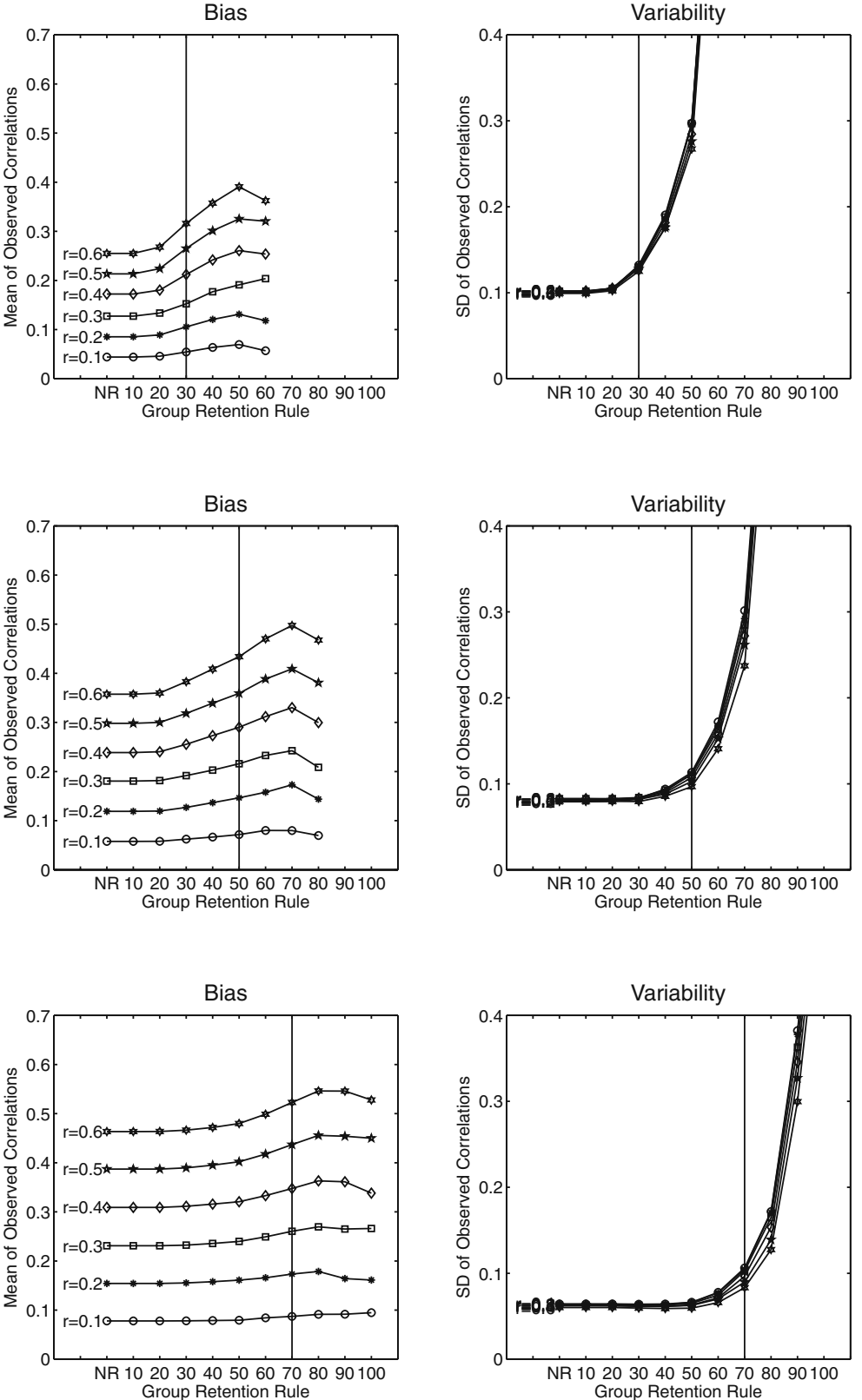

Group Retention Rule
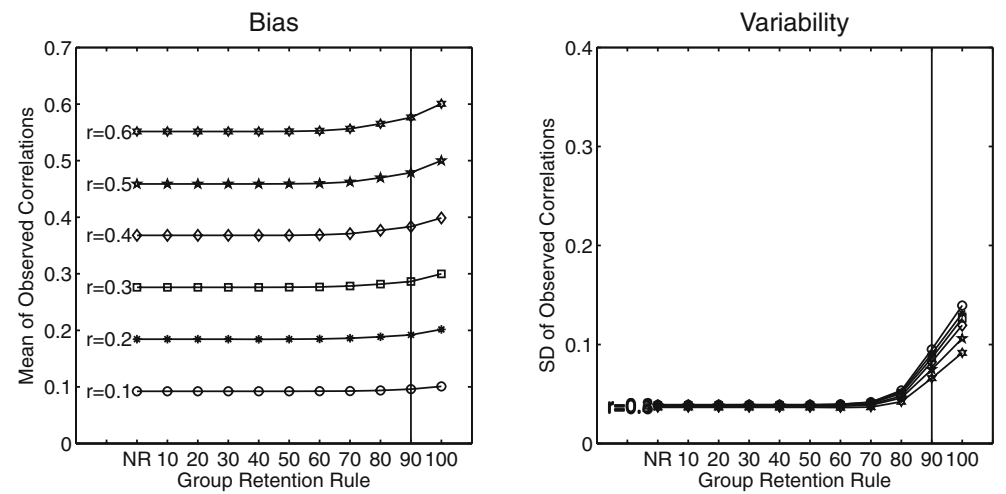

Fig. 3 Study 2: linear nonresponse. Mean absolute error, bias, and variability for each retention rule across various response rates 


\section{0\% Response Rate}

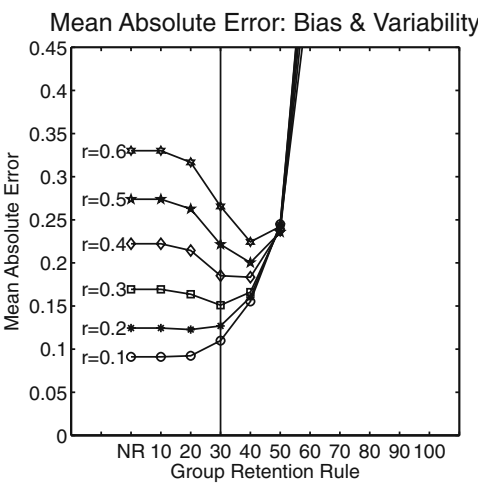

\section{$50 \%$ Response Rate}

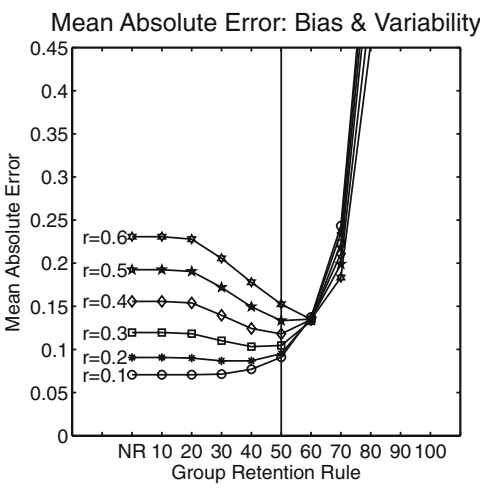

\section{0\% Response Rate}

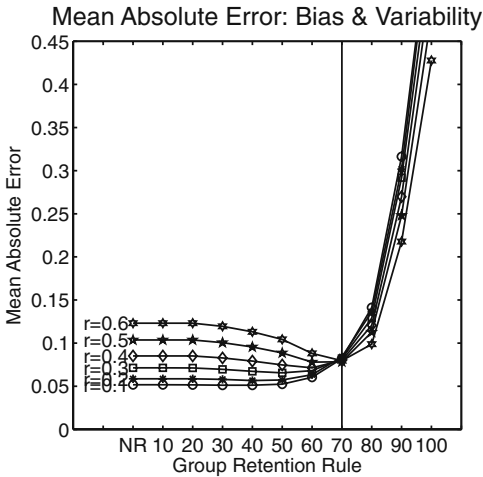

\section{0\% Response Rate}

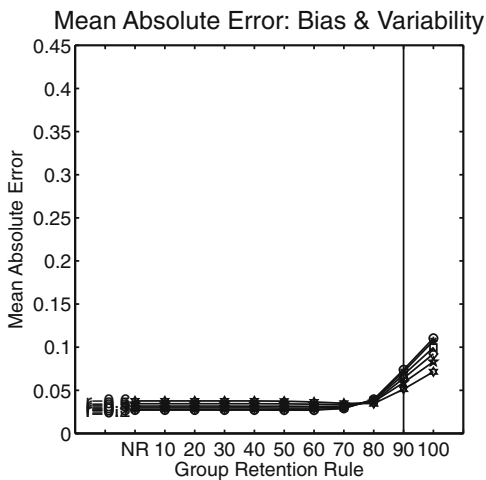

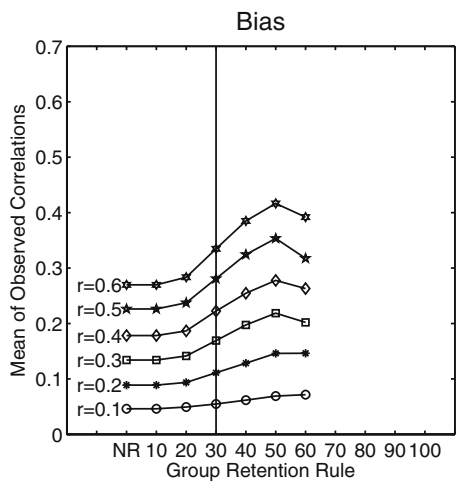
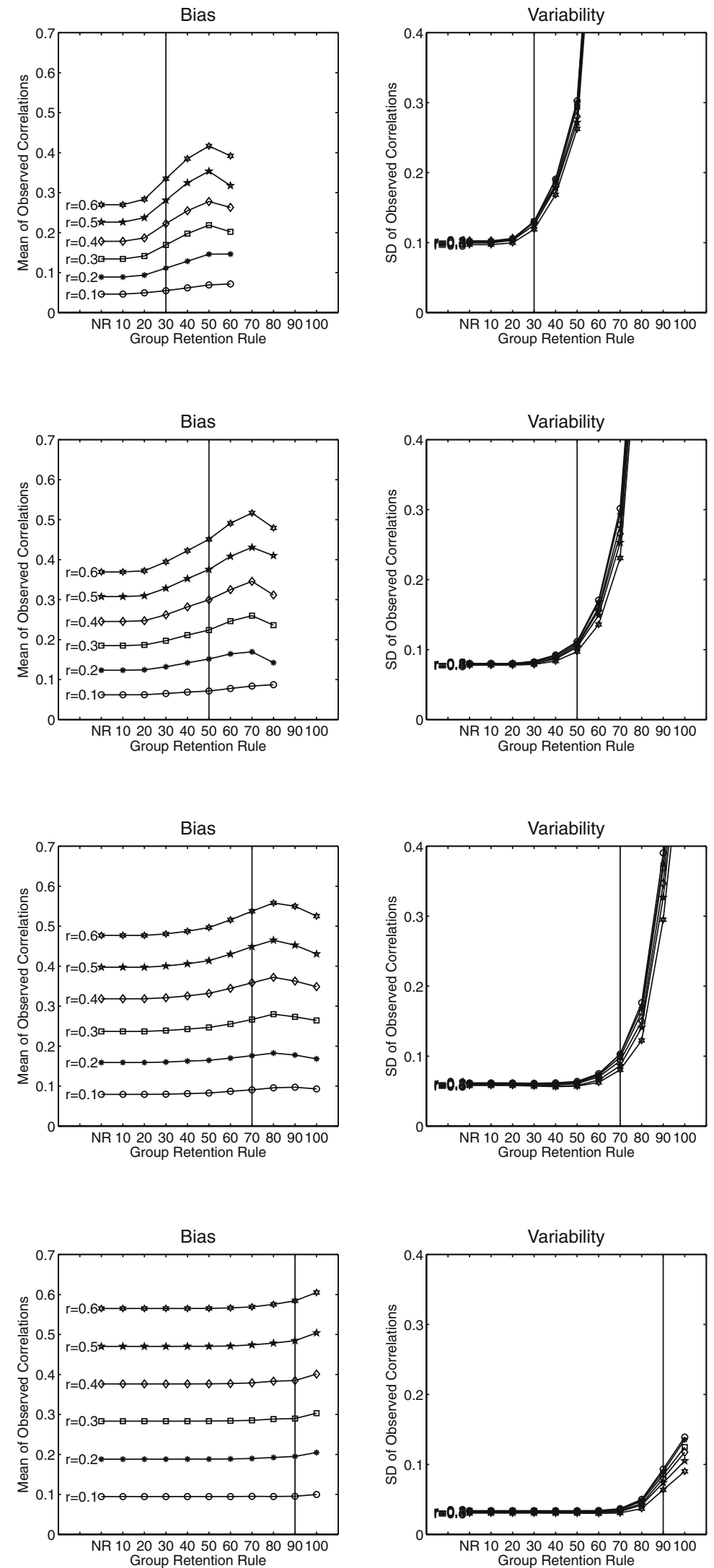

Fig. 4 Study 2: linear nonresponse (within-group one-tail). Mean absolute error, bias, and variability for each retention rule across various response rates 
Close inspection of our graphs reveals that data that are missing systematically do influence the accuracy of estimates of diversity relations. There were differences in the levels of mean absolute error across the various forms of nonresponse. Overall, however, the two types of systematic nonresponse that we modeled produced a pattern of results, with respect to the impact of group-retention rules, that was extraordinarily similar to random nonresponse. ${ }^{5}$ This suggests that in the diversity context, retention rules are inappropriate for both random and systematic missing data.

\section{General discussion}

In recent years, considerable attention has been devoted to determining the extent to which within-group diversity is related to a variety of group-level processes and outcomes. Unfortunately, the individual-level information used to calculate group diversity indices is often collected using surveys with less-than-perfect response rates. As a consequence, the resulting diversity-outcome correlations observed in this literature are influenced not only by the "true" underlying relations among the variables but also by the impact, on the diversity measures, caused by the missing data.

Previously, Allen et al. (2007b) examined the magnitude of impact of participant nonresponse on observed diversity relations for continuous variables using a series of computer simulations. Further, they noted that many researchers used group-retention rules that were based on within-group response rates. In the present study, we examined the effectiveness of these group-retention rules for ameliorating the impact of participant nonresponse on observed diversity relations. The most important observation from our investigation is that the impact of a particular group-retention rule depended on the strength of the true correlation being estimated and the response rate. Specifically, we found that group-retention rules were not able to compensate for a low response rate. That is, using a groupretention rule did not increase the accuracy of estimates obtained at one response rate (e.g., 50\%) to be equal to those obtained a higher response rate (e.g., $70 \%$ or $90 \%$ ). That said, we found that within a given response rate, group-retention rules could influence the accuracy of estimates of relations (often, negatively).

\section{Limitations}

Our research must be considered in the context of potential limitations. First, we did not simulate every possible

\footnotetext{
$\overline{5}$ This overall pattern of results was the same as well for the two nonlinear nonresponse simulations (i.e., within-group two-tail, and within-group median) referred to in footnote 3 .
}

nonresponse scenario that a diversity researcher might encounter. Rather, we asked the "what happens if . . . ?" question for a number of different missing-data scenarios. Moreover, our results do not address the question of which nonresponse patterns actually occur in organizational surveys. Theorizing and empirical data do suggest, however, that the nonresponse patterns that we modeled in our simulations are not unreasonable (see Allen et al., 2007a; Borg \& Baumgärtner, 2008; Rogelberg et al., 2000, 2003; Williams, Allen, \& Carter, 2010). Nonetheless, nonresponse can occur in countless ways, and we could not model them all. Second, our investigation was limited to the effect of nonresponse when group diversity was conceptualized with respect to continuous variables. It may be that group-retention rule recommendations would be different for categorical data. Finally, we certainly acknowledge that there may be boundary conditions that moderate our findings. For example, Timmerman (2005) describes potential boundary conditions for the impact of missing data in a group context.

\section{Recommendations for researchers}

Ultimately, it must be emphasized, of course, that the best situation is one in which complete data are available from every group that is involved in a given study. Indeed, our simulation results clearly illustrated that group-retention rules are no substitute for a high response rate. Specifically, we found that in no circumstances did group-retention rules cause the accuracy of estimates obtained at a particular response rate to approximate the accuracy of estimates obtained at a higher response rate. Therefore, we strongly advise researchers to "go the extra mile" to obtain a high response rate. In this regard, numerous authors have described strategies for obtaining higher response rates (e.g., Dillman, 2007; Fox, Crask, \& Kim, 1988). In calling for the use of complete groups in diversity research, however, we recognize that this is not always possible. Consequently, in situations with less than perfect response rates, researchers may wonder how to proceed with their analyses. Should a group-retention rule be used? If so, what should it be?

Our simulations revealed that in circumstances relevant to diversity researchers (i.e., those in which the true correlation is less than $r=.40$ ), retention rules will not increase the accuracy of estimates of diversity-outcome relations. Moreover, on average, the accuracy of estimates of diversity-outcome relations will often decrease if retention rules are applied. This pattern of results was consistent across the various types of random and systematic nonresponse that we modeled.

Interestingly, our simulations did show an increase in the accuracy of estimates when the true correlation was $r=.40$ or higher and the response rate was low. In these circum- 
stances, using a group-retention rule equal to (or slightly above) the response rate resulted in more accurate observed correlations. Because true correlations .40 or above are not typical in diversity research, however, we urge diversity researchers not to focus on this aspect of our results. Moreover, we must stress that even though increases in accuracy could be obtained with strong true correlations, those increases came at a high price. Specifically, the increase in accuracy was maximized when approximately $50 \%$ of groups were dropped. Thus, even under an "optimal" retention rule, a sufficient number of groups will be dropped such that statistical power will decrease substantially. This decrease in statistical power is a frequent concern with deletion approaches to missing data (Switzer \& Roth, 2002). Moreover, deleting groups violates what Newman (2009) refers to as "a simple yet fundamental principle" (p. 11) of missing-data analysis: use all of the available data.

Researchers may wonder what the consequences of our findings are for interpreting existing research. In the typical diversity scenario (i.e., true $r<.40$ ), our simulations illustrated that dropping groups (via retention rules) decreased the accuracy of observed correlations (due to the variability in observed correlations created by dropping groups). This means that the correlations observed in the existing research may underestimate or overestimate the true correlation when diversity rules are used. Consequently, we would have the most confidence when interpreting findings from studies that did not use retention rules.

When researchers do use retention rules, how should their findings be interpreted? Based on our results, we would have the most confidence in findings from studies with very low retention rules (i.e., closest to the state where retention rules are not used). Indeed, to minimize the reduction in accuracy created by retention rules, the retention rule should be at least $10 \%$ to $20 \%$ lower than the response rate (e.g., with a $70 \%$ response rate, we suggest using a retention rule of $60 \%$ or less). Again, though, our preference is for studies that did not use retention rules. In contrast, our findings suggest that studies that used a group-retention rule greater than the overall response rate have findings that are highly suspect and possibly misleading.

In summary, higher response rates lead to more accurate study findings - regardless of the group-retention rule used. We strongly encourage researchers to do everything in their power to maximize the response rates in their investigations. Group-retention rules are not substitutes for achieving high response rates. Indeed, in most of the circumstances that we modeled that were relevant to diversity researchers, the use of retention rules had little impact on the accuracy of relations, or actually decreased the accuracy of relations. In extremely limited circumstances (i.e., low response rates and high true correlations) group-retention rules can improve accuracy slightly - but at a great price (a substantial loss of statistical power). Consequently, we urge diversity researchers to discontinue the use of retention rules.

\section{Conclusions}

Diversity researchers are, from time to time, blessed with a $100 \%$ response rate. More typically, however, they must contend with the problem of participant nonresponse. In the diversity literature, a common approach is to apply some sort of group-retention rule to deal with the problem of participant nonresponse. Our research is, to the best of our knowledge, the first to examine empirically the influence of group-retention rules within this literature. The results of our simulations suggest that, in the majority of scenarios in which diversity researchers might like to apply retention rules (i.e., scenarios with a true correlation of less than $r=$ .40 ), these rules will either have little impact on, or decrease the accuracy of, estimates of relations. Consequently, we suggest that group researchers discontinue the use of groupretention rules and instead focus on strategies for ensuring high response rates.

Author Note Support for this research was provided by the Social Sciences and Humanities Council of Canada (Grant 410-2009-2095 awarded to D.J.S.; Grant 410-2007-0742 awarded to N.J.A. and H.M.W.).

\section{References}

Allen, N. J., Stanley, D. J., Williams, H., \& Ross, S. J. (2007a). Assessing dissimilarity relations under missing data conditions: Evidence from computer simulations. The Journal of Applied Psychology, 92, 1414-1426.

Allen, N. J., Stanley, D. J., Williams, H., \& Ross, S. J. (2007b). Assessing the impact of non-response on work group diversity effects. Organizational Research Methods, 10, 262-286.

Allison, P. D. (2002). Missing data. Thousand Oaks: Sage.

Ancona, D., \& Caldwell, D. (1992). Demography and design: Predictors of new product team performance. Organization Science, 3, 321-341.

Borg, I. \& Baumgärtner, M. K. (2008). Does demographic item nonresponse cause biased results in employee surveys? Poster presented at the annual meeting of the Society for Industrial and Organizational Psychology, San Francisco, CA.

Byrne, D. (1971). The attraction paradigm. New York: Academic Press.

De Dreu, C. K. W., \& West, M. A. (2001). Minority dissent and team innovation: The importance of participation in decision-making. The Journal of Applied Psychology, 86, 1191-1201.

Dekking, F. M., Kraaikamp, C., Lopuhaa, H. P., \& Meester, L. E. (2005). A modern introduction to probability and statistics. Delft: Springer.

Dillman, D. A. (2007). Mail and Internet surveys: The tailored design method -2007 update with new Internet, visual, and mixed-mode guide (2nd ed.). Hoboken: Wiley. 
Fox, R. J., Crask, M. R., \& Kim, J. (1988). Mail survey response rate: A meta-analysis of selected techniques for inducing response. Public Opinion Quarterly, 52, 467-491.

Gruenfeld, D. H., Mannix, E. A., Williams, K. Y., \& Neale, M. A. (1996). Group composition and decision making: How team member familiarity and information distribution affect process and performance. Organizational and Human Decision Processes, $67,1-15$.

Hackman, J. R. (1992). Group influences on individuals in organizations. In M. D. Dunnette \& L. M. Hough (Eds.), Handbook of industrial and organizational psychology (Vol. 3, pp. 199-268). Palo Alto: Consulting Psychologists Press.

Harrison, D. A., \& Klein, K. J. (2007). What's the difference? Diversity constructs as separation, variety, or disparity in organizations. Academy of Management Review, 32, 1199-1228.

Hogg, M., \& Abrams, D. (1988). Social identification. London: Routledge.

Horwitz, S. K., \& Horwitz, I. B. (2007). The effects of team diversity on team outcomes: A meta-analytic review of team demography. Journal of Management, 33, 987-1015.

Jackson, S. E., Brett, J. F., Sessa, V. I., Cooper, D. M., Julin, J. A., \& Peyronin, K. (1991). Some differences make a difference: Individual dissimilarity and group heterogeneity as correlates of recruitment, promotions, and turnover. The Journal of Applied Psychology, 76, 675-689.

Jehn, K. A., Northcraft, G. B., \& Neale, M. A. (1999). Why differences make a difference: A field study of diversity, conflict, and performance in workgroups. Administrative Science Quarterly, 44, 741-763.

King, E. G., Hebl, M. R., \& Beal, D. J. (2009). Conflict and cooperation in diverse workgroups. Journal of Social Issues, 2, 261-285.

Liao, H., Joshi, A., \& Chuang, A. (2004). Sticking out like a sore thumb: Employee dissimilarity and deviance at work. Personnel Psychology, 57, 969-1000.

Little, R. J. A., \& Rubin, D. B. (2002). Statistical analyses with missing data. New York: Wiley.

Maloney, M. M., Johnson, S. G., \& Zellmer-Bruhn, M. E. (2010). Assessing group-level constructs under missing data conditions: A Monte Carlo simulation. Small Group Research, 41, 281-307.

Mannix, E., \& Neale, M. A. (2005). What differences make a difference? The promise and reality of diverse teams in organizations. Psychological Science in the Public Interest, 6 , $31-55$.

Mayo, M., Pastor, J. C., \& Meindl, J. R. (1996). The effects of group heterogeneity on the self-perceived efficacy of group leaders. Leadership Quarterly, 7, 265-284.

McKnight, P. E., McKnight, K. M., Sidani, S., \& Figueredo, A. J. (2007). Missing data: A gentle introduction. New York: Guilford Press.

Newman, D. A. (2003). Longitudinal modeling with randomly and systematically missing data: A simulation of ad hoc, maximum likelihood, and multiple imputation techniques. Organizational Research Methods, 6, 328-362.

Newman, D. A. (2009). Missing data techniques and low response rates: The role of systematic nonresponse patterns. In C. E. Lance \& R. J. Vandenberg (Eds.), Statistical and methodological myths and urban legends (pp. 7-36). New York: Routledge.
Newman, D. A., \& Sin, H. P. (2009). How do missing data bias estimates of within-group agreement? Sensitivity of $\mathrm{SD}_{\mathrm{WG}}$,

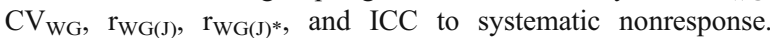
Organizational Research Methods, 12, 113-147.

O'Reilly, C. A., Caldwell, D. F., \& Barnett, W. P. (1989). Work group demography, social integration, and turnover. Administrative Science Quarterly, 34, 21-37.

Reagans, R., Zuckerman, E., \& McEvily, B. (2004). How to make the team: Social networks vs. demography as criteria for designing effective teams. Administrative Science Quarterly, 49, 101-133.

Riordan, C. M. (2001). Relational demography within groups: Past developments, contradictions, and new directions. Research in Personnel and Human Resource Management, 19, 131-173.

Rogelberg, S. G., Conway, J. M., Sederburg, M. E., Spitzmüller, C., Aziz, S., \& Knight, W. E. (2003). Profiling active and passive nonrespondents to an organizational survey. The Journal of Applied Psychology, 88, 1104-1114.

Rogelberg, S. G., Luong, A., Sederburg, M. E., \& Cristol, D. S. (2000). Employee attitude surveys: Examining the attitudes of noncompliant employees. The Journal of Applied Psychology, $85,284-293$.

Roth, P. L. (1994). Missing data: A conceptual review for applied psychologists. Personnel Psychology, 46, 537-560.

Roth, P. L., Switzer, F. S., \& Switzer, D. M. (1999). Missing data in multiple item scales: A Monte Carlo analysis of missing data techniques. Organizational Research Methods, 2, 211-232.

Rubin, D. (1976). Inference and missing data. Biometrika, 63, 581-592.

Schafer, J. L., \& Graham, J. W. (2002). Missing data: Our view of the state of the art. Psychological Methods, 7, 147-177.

Schippers, M. C., den Hartog, D. N., Koopman, P. L., \& Wienk, J. A. (2003). Diversity and team outcomes: The moderating effects of outcome interdependence and group longevity and the mediating effect of reflexivity. Journal of Organizational Behavior, 24, 779-802.

Spearman, C. (1910). Correlation calculated with faulty data. British Journal of Psychology, 3, 271-295.

Switzer, F. S., \& Roth, P. L. (2002). Coping with missing data. In S. G. Rogelberg (Ed.), Handbook of research methods in industrial and organizational psychology (pp. 310-323). Malden: Blackwell.

Switzer, F. S., Roth, P. L., \& Switzer, D. M. (1998). Systematic data loss in HRM settings: A Monte Carlo analysis. Journal of Management, 24, 763-779.

Tajfel, H., \& Turner, J. (1986). The social identity of intergroup behavior. In S. Worchel \& W. Austin (Eds.), Psychology and intergroup relations (pp. 7-24). Chicago: Nelson-Hall.

Timmerman, T. A. (2005). Missing persons in the study of groups. Journal of Organizational Behavior, 26, 21-36.

van Knippenberg, D., De Dreu, C. K. W., \& Homan, A. C. (2004). Work group diversity and group performance: An integrative model and research agenda. The Journal of Applied Psychology, 89, 1008-1022.

Williams, H. M., Allen, N. J., \& Carter, A. J. (2010). Will I respond to the organization's survey? Depends who I work with! Presented at the 2nd Biennial Institute of Work Psychology Conference on Work, Well-Being and Performance, Sheffield, U.K.

Williams, K. Y., \& O'Reilly, C. A. (1998). Demography and diversity in organizations: A review of 40 years of research. Research in Organizational Behavior, 20, 77-140. 